\title{
Evolving legged robots using biologically inspired optimization strategies
}

\author{
Beatrice G.R. Smith, Dr. Chakravarthini M. Saaj, and Dr. Elie Allouis
}

\begin{abstract}
When designing a legged robot a small change in one variable can have a significant effect on a number of the robot's characteristics, meaning that making tradeoffs can be difficult. The algorithm presented in this paper uses biologically inspired optimization techniques to identify the effects of changing various robot design variables and determine if there are any general rules which can be applied to the design of a legged robot. Designs produced by this simulation are also compared to existing robot designs and biological systems, showing that the algorithm produces results which require less power than other robots of a similar mass, and which share a number of characteristics with biological systems.
\end{abstract}

\section{INTRODUCTION}

The degree to which biological inspiration is used in robotics varies greatly, from very abstracted principles such as those which inspired Rhex [1], to systems such as the Lobster robot [2] which combine biologically inspired morphology, control and actuators to be as close to nature as possible. However, a biological system has many extra constraints that a robot does not have, both in terms of materials and function, so copying an animal as closely as possible will not produce an efficient robot design. In many cases different aspects of animal morphology complement each other. If a single feature is used without the complementary features it may not work as well as it does on the actual organism.

One method of deciding how far to copy a biological system is to carry out extensive studies on the organism in question, often with the help of biologists. Examples of this approach and its impressive results can be seen in [1] and [2]. However this may be unrealistic for researchers who do not have the time, facilities or expertise to conduct biological experiments, and papers published in biological journals do not always concentrate on the specific attributes of animals that engineers are interested in. Luckily it is evident that there are certain 'design features' which are common throughout the animal kingdom, for example the fact that regardless of number of legs, locomotion can be modeled as an inverted pendulum, or similarities in posture between animals of similar sizes.

Evolutionary algorithms are algorithms which simulate the biological process of evolution; a population of individuals is created, and these can be combined (mated) or randomly changed (mutated) to create new individuals. A

Manuscript received July 22, 2010. This work was supported in part by Astrium UK and the EPSRC.

B. G. R. Smith is with the Surrey Space Centre, University of Surrey, UK (Phone: (+44)1483-682224; Fax: (+44)1483-689503; e-mail:

B.G.Smith@surrey.ac.uk).

Dr. C. M. Saaj is with the Surrey Space Centre, University of Surrey, UK (e-mail: C.Saaj@surrey.ac.uk).

Dr. E. Allouis is with Astrium UK, Stevenage (e-mail: elie.allouis@astrium.eads.net). cost function is used to assess the fitness of each individual, and individuals with low fitness (i.e. a high cost) are removed from the population, resulting in an overall optimization of the population. Evolutionary programming has been used in a number of areas in robotics, and is often combined with other AI techniques such as fuzzy logic to produce evolving control systems, for example for producing optimal gaits [4], however much less work has been done on evolving robot morphology. Projects which have concentrated on this area either tend to have the basic design of the robot already decided and are using the evolutionary algorithm to optimize this initial design [5], or concern the design of a virtual system and therefore real world constraints such as power consumption are not considered [6]. This paper presents an algorithm using evolutionary algorithms to produce legged vehicle designs which in many cases are similar to biological systems; however they are optimized as mechanical systems. The paper is organized as follows: Section II details the development and implementation of the algorithm, Section III analyses some results of the algorithm and identifies some design rules for legged robots, and Section IV gives a conclusion and suggestions for further work.

\section{DESIGN AND IMPLEMENTATION}

As described above, evolutionary algorithms have already been used to produce specific robot characteristics; for example walking gaits. However unlike previous works this paper takes the approach that the robot should be considered as a complete system. This is for two reasons; firstly, biological systems evolve as complete systems, secondly there is a high degree of coupling between the variables which make up the robot configuration. A technique which follows the biological strategy and takes the coupling into account is likely to be more successful than one which neglects to consider the effect of changing one aspect of the system on the other aspects. The algorithm is made up of three modules; the first is a genetic algorithm which produces a population of robot designs, and then optimizes these, the best result after fifty iterations is added to a second population. This is done so that the results in the second population go some way to fulfill the criteria described by the weighting in the cost function, yet still have a degree of variation. The genetic algorithm is repeated until the second population is made up of twenty individuals. The second population is further optimized using extremal optimization, and then the best result is selected using Pareto optimization.

\section{A. Genetic Algorithm}

Since one of the areas being investigated with this algorithm was whether there are any general rules which can be used in the design of legged robots, the algorithm was given a high number of variables to work with, as well as 
TABLE I

VARIABLES USED IN GENETIC ALGORITHM

\begin{tabular}{|c|c|c|}
\hline Variable & Minimum value & Maximum value \\
\hline Body length & Payload length & Payload length $\times 2$ \\
\hline Body Width & Payload width & Payload width x 2 \\
\hline Body height & Payload height & Payload height $\times 1.5$ \\
\hline Leg segment 1 length & $0 *$ & $0.5 \mathrm{~m}$ \\
\hline Leg segment 2 length & $0 *$ & $0.5 \mathrm{~m}$ \\
\hline Leg segment 3 length & $0 *$ & $0.5 \mathrm{~m}$ \\
\hline Leg segment 4 length & $0 *$ & $0.5 \mathrm{~m}$ \\
\hline Leg diameter & 0 & $25 \%$ \\
\hline Number of legs & 4 & 8 \\
\hline $\begin{array}{l}\% \text { of legs on ground } \\
\text { in stance phase }\end{array}$ & $35 \%$ & $85 \%$ \\
\hline Tail & 0 (Boolean) & 1 (Boolean) \\
\hline Joint & 0 (Boolean) & 1 (Boolean) \\
\hline
\end{tabular}

wide ranges for these variables. This would mean that there would be a good variety of different robot designs produced by the genetic algorithm. The variables which made up a robot design (referred to as a chromosome in this paper), and their possible ranges, are detailed in Table 1.

These variables allow the genetic algorithm to vary the dimensions of the legs and body, add or remove a tail or a body joint, and change the gait of the robot. One criterion for the algorithm was that the designs it produced should be feasible for building real, useful robots. For this reason the user is able to specify a payload mass and dimensions, which the algorithm will take into account when designing the robot. Additionally the user is able to set a desired mass range and construction material for the robot, and adjust the weights of the factors in the cost function. This is particularly important as the emphases of the robot's design are very much dependent on the robot's mission. For example a robot for search and rescue would need to be fast and agile, while power could be supplied via a tether so high torque motors could be used, however a robot for planetary exploration would not have this option and therefore minimizing torque and power requirements would be a much more important design factor. The user can therefore adjust the weights of the torque, speed, workspace, stability and mobility of the robot. The outline of the genetic algorithm is as follows:

1) Initialize population (POP1) of fifty chromosomes

2) Find cost of each chromosome

3) Select chromosomes to mate - this is done by ordering the chromosomes by cost and then choosing two random numbers on a Gaussian distribution. This ensures that there is a greater likelihood of lower cost chromosomes being chosen

4) Mate chromosomes (a single crossover point is used)

5) Mutate the population by randomly selecting a variable in a chromosome and incrementing it by a variable $\sigma$ which changes with population size. The number of mutations which occur depends on the size of the population, so that the greater the population, the fewer mutations will occur. This was selected since as the average cost of the population decreases members are less likely to be removed, thus the population size will increase. Mutations are therefore more likely to be detrimental rather than beneficial.

6) Recalculate costs for each chromosome

7) Remove chromosomes with higher than average costs from the population

8) Return to 3

The cost function is calculated using the following equation:

$$
\operatorname{Cost}=a^{*} \tau-\left(b^{*} v+c^{*} w+d^{*} s+e^{*} m\right)+25 * \partial m
$$

Where $a, b, c, d, e$ are the user defined weights for torque, speed, workspace, stability and mobility respectively and $\tau$, $v, w, s, m$ are the calculated values for torque, speed, workspace, stability and mobility respectively. $\partial \mathrm{m}$ is the difference between the current mass and the desired mass range, and is multiplied by 25 to make sure it overrides the other components. Using this equation means that high values of torque increase the cost, while high values of speed, workspace, stability and mobility decrease the cost.

The robot is modeled as a collection of robotic arms supporting a mass (the robot chassis). This allows equations usually used to calculate manipulator torques and workspace to be used to calculate values for the legged vehicle [7], [8]. It is assumed that the robot would move using a statically stable gait, therefore Waldron's equation for maximum speed can be used [9], and a measure of stability can be calculated by adding the stability margin [10] to the maximum angle of slope the robot could ascend before tipping over. Mobility is calculated using the simplified Kutzback equation [11].

Once the population has achieved an average cost lower than the target, or more than fifty generations have passed, the chromosomes are ordered according to their cost, and the best one is added to the second population (POP2). This occurs twenty times until POP2 is filled.

\section{B. Extremal optimization}

Once POP2 has been filled it goes through extremal optimization. This technique was inspired by self organization in nature [12], and unlike the genetic algorithm requires a predefined input to work on, which in this case is provided by the chromosomes which make up POP2. Extremal optimization was selected in this case as it has better performance at overcoming local minima than alternative methods such as simulated annealing. This is desirable since if the algorithm is trapped in a local minimum it will not be able to optimize any further and find the true minimum. Avoiding this is particularly important in the case of this algorithm as the high number of variations that are possible results in a strong possibility of local minima. The extremal optimizer works by identifying the variable in a chromosome which has the greatest negative effect, then changing this value to improve the overall cost. 
This is carried out as follows:

1) For each variable in a chromosome, increment the variable by a random number while keeping the rest of the variables the same as in the original chromosome, then recalculate the cost of the chromosome

2) Order the variables according to the difference between the cost of the chromosome with the changed variable and the original

3) Select a value on a probability distribution, such that there is the greatest probability of selecting 1 , and the lowest probability of selecting 12 .

4) For the corresponding variable in the chromosome, change the value to a random number within the limits of the variable

5) Recalculate cost and compare to the original cost

6) If cost has improved, replace the original chromosome with the new one.

The optimization was then repeated 10,000 times for each chromosome, this number was selected based on the number of iterations it would take the algorithm to converge to a final result.

\section{Pareto optimization}

It was found that the algorithm was able to produce optimized results which had characteristics following the input weights. However, the results of repeated runs often diverge significantly due to the high number of combinations of body and leg dimensions. Because of this Pareto optimization is used to differentiate between a number of competing designs. This was done by calculating the values for each of the cost function components for each chromosome, and comparing this to the values for that component for the other chromosomes. If the chromosome had a superior value (e.g. the lowest torque, or highest speed) then its score would be incremented with a value equal to the weight of that component. This was carried out for all the chromosomes, with the chromosome with the highest overall score being selected as the best result.

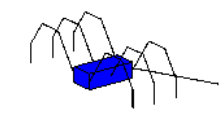

Mass $=4.3259 \mathrm{~kg}$
Torque in $\mathrm{J} 1=1.0722 \mathrm{e}-016 \mathrm{Nm}$ Torque in $\mathrm{J} 2=2.1564 \mathrm{Nm}$ with 3 legs on the ground Total torque $=13.9348 \mathrm{Nm}$ Workspace $=0.097792 \mathrm{m3}$ Stability $=2.5987$

Peak Power $=6.8615$
There is no body joint Torque in $\sqrt{3}=2.4885 \mathrm{Nm}$ Total toraue per lea $=4.6449 \mathrm{Nm}$ Maximum speed $=0.028165 \mathrm{~m} / \mathrm{s}$

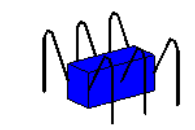
Mass $=27.943 \mathrm{~kg}$
Toraue in $\mathrm{J1}=1.8621 \mathrm{e}-015 \mathrm{Nm}$ $\begin{aligned} \text { Torque in } \sqrt{2} & =4.5887 \mathrm{Nm} \\ \text { Torque in } \sqrt{3} & =6.4549 \mathrm{Nm}\end{aligned}$ Total torque per leg $=11.0436 \mathrm{Nm}$ with 3 legs on the ground Total torque $=33.1307 \mathrm{Nm}$ Maximum speed $=0.049497 \mathrm{~m} / \mathrm{s}$ Workspace $=0.09248 \mathrm{m3}$ Stability $=1.7799$ Peak Power $=12.9082 \mathrm{~W}$ There is no body joint

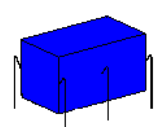

Mass $=102.6868 \mathrm{~kg}$ Torque in $\mathrm{J} 1=4.8573 \mathrm{e}-015 \mathrm{Nm}$ Torque in $\mathrm{J} 2=2.2119 \mathrm{Nm}$ Torque in $\mathrm{J} 3=8.083 \mathrm{Nm}$ Total torque per leg $=10.2949 \mathrm{~N}$ with 4 legs on the ground Total torque $=41.1797 \mathrm{Nm}$ Workspace $=0.023352 \mathrm{m3}$ Stability $=1.7164$ Peak Power $=15.4436$ There is no body joint Maximum speed $=0.038178$

Fig. 1. Example outputs of evolutionary program. These correspond to EP1, EP4 and EP5 in Table 2.
Finally, the algorithm displayed a sketch of the optimal design, along with its characteristics. Examples of some of the results produced are shown in Fig. 1.

\section{RESULTS AND ANALYSIS}

\section{A. Trends in designs}

Three main areas were investigated to determine if any trends could be found in the designs produced by the algorithm. These were; ratio of body length to body width, ratio of leg length to body length, and the relative lengths of each of the leg segments. The ratios were used so as to ensure the trends were not specific to a particular body shape or robot mass.

Fig. 2 shows how the dimensions of the robot's body change as the priorities of the user change. As torque becomes more important, there is an overall reduction in the ratio of body length to width, ending up at a ratio of 1.23:1. Since the length: width ratio of the payload in this case was $1.33: 1$, this suggests that a more square body shape might be an advantage when attempting to reduce torque. This could be due to the fact that this minimizes the body size while maintaining speed, stability and workspace. This possibility is corroborated by the fact that increasing workspace weight causes a decrease in body length: width ratio, since the smaller the body is the longer the legs can be for a given mass. Additionally the change in ratio due to stability weighting almost mirrors that due to torque. A larger body spreads the legs out further and therefore increases stability, however it also increases the torque required. On the other hand, as speed becomes more important the ratio of length: width increases to 1.92 . This is because the greater the distance between the legs the faster the robot can move, so a long body allows more room between legs, for a given number of legs.

Fig. 3 shows to changing th obvious result increases the le

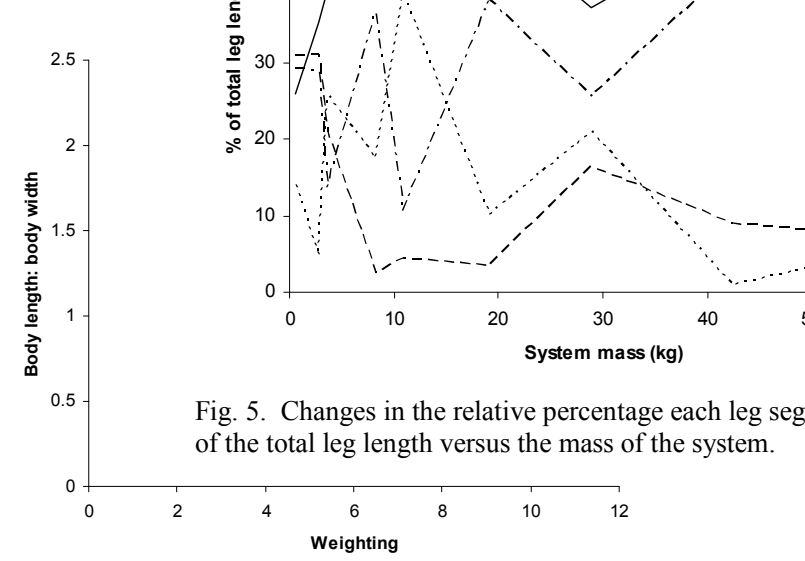

Fig. 2. Changes in body length: width ratio as the weights of the various cost function components increase. 


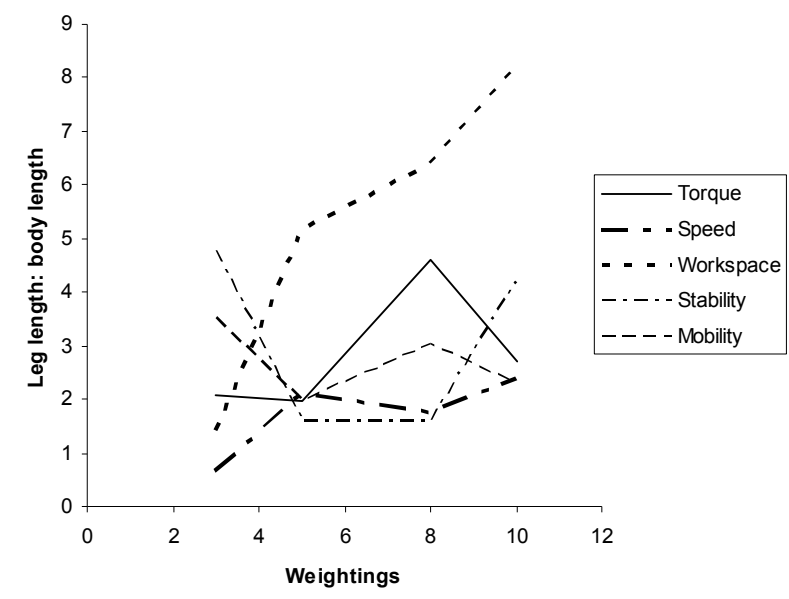

Fig. 3. Changes in ratio of leg length: body length as the weights of the various cost function components increase.

length of the body, this is unsurprising since it means that for a given mass a greater proportion can be made up of leg rather than body. Similarly, as torque weighting increases, the body becomes smaller so the ratio of leg length: body length also shows an overall increase. Mobility and speed both converge on 2.00 , since neither of these depend on leg length, it is possible that a 2:1 leg: body length is the value that the algorithm will converge to when no one factor is dominant. It was expected that as stability weighting increased the leg: body length would also increase, however there in a dip when intermediate values of weightings are used. It is possible that at these values stability is more closely related to body length; the fact that the dip in Fig. 3 corresponds to a peak in Fig. 2 supports this.

Fig. 4 shows how the relative lengths of the leg segments change with torque weighting. In all cases segments 1 and 4 make up the majority of the leg, while the middle segments, 2 and 3, stay small. Eventually segments 2 and 3 converge to approximately the same proportions. There appears to be some relationship between segments 4 and 3, and 2 and 1 , since as one increases the other decreases, and vice versa. The main reason for this is that the smaller the horizontal distance between the foot and the body the lower the torque required to support the robot, and since the angles of the leg

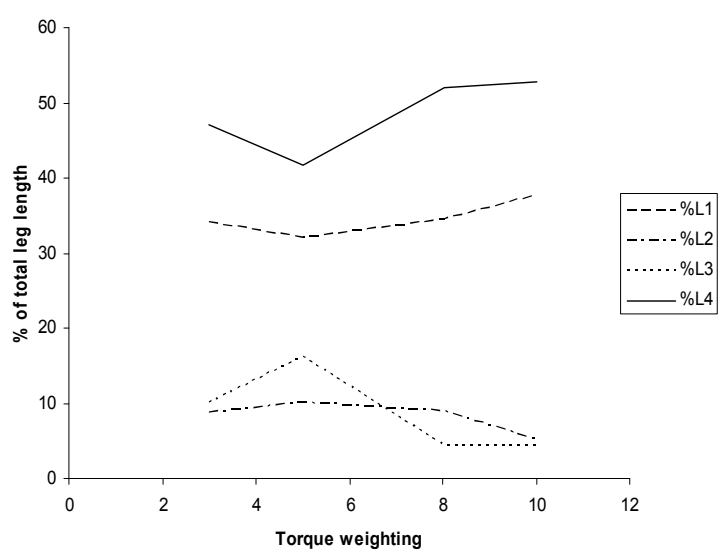

Fig. 4. Changes in the relative percentage each leg segment makes up of the total leg length versus the torque weighting.

segments cannot been changed the lengths of the more horizontal leg segments must be minimized. Since segment 4 can only move horizontally it does not contribute to the vertical torque. This means that it does not have to be minimized, and explains why it is the longest segment. A similar trend can be seen in Fig. 5, where once again segments 1 and 4 are the longest. Fig. 6 shows that the changes in the relative percentages of the different leg segments due to workspace are very different. In this case, the segment percentages start with segments 2 and 3 as the smallest, and segment 4 being the longest; however as the weighting of workspace increases the segment lengths start to converge, until they end up being equal at around $25 \%$. This suggests that for maximum workspace for a given leg length all the leg segments should be the same length.

In terms of general rules for legged robot design, it appears that a square, or near square body might be preferable, as this would minimize mass. However, the number of legs will have to be selected so as to ensure the speed of the vehicle is not too low. The number of legs on the vehicle is related to the length of the vehicle; if the chassis is short (for example due to torque being minimized), or speed is a priority there tends to be fewer legs so as to ensure the maximum speed is not too low. Conversely if speed is a very low priority or if the body is long and thin the number of legs tends to be eight, since this improves stability and system mobility. In some cases when torque is extremely dominant and the body is very short there will only be 4 legs, as this reduces the mass of the robot. In order to improve stability the effective length of the body should be increased, either by adding a tail or by designing the legs such that they are able to lengthen the rover's footprint on steep slopes. In terms of leg dimensions it appears that the biggest reduction in torque comes from reducing the lengths of segments 2 and 3; however this could adversely affect the stability and workspace. Therefore having each segment the same length, as is the case when maximizing workspace, may be preferable. Long legs are an advantage for a high workspace, and high stability, however shorter legs are preferred when torque must be minimized as much as possible. Additionally, if torque is the dominant

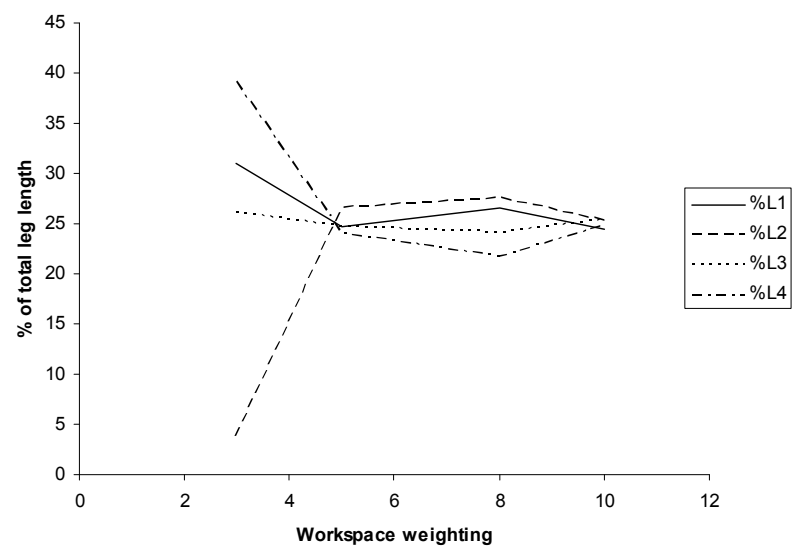

Fig. 6. Changes in the relative percentage each leg segment makes up of the total leg length versus the workspace weighting.

factor some of the leg segment lengths may reduce so far 
that the leg loses a degree of freedom, but four degrees of freedom are preferred for high mobility and workspace.

B.Comparison with existing robotic and biological systems

Five evolutionary algorithm results (EP1-5) were compared to a number of existing walking robot systems, and to a range of animal phyla. The masses of the generated robot designs were selected to be similar to those of the existing systems, and the payload masses were selected to be $20 \%$ of the total system mass. This is a typical value for legged robots, however is above average for planetary rovers [13]. Values for dimensions and masses for the existing systems have been taken from literature where possible, however since many legged systems are technology demonstrators rather than designed for specific missions data for payload capacity is limited. The evolutionary algorithm results were also compared against a range of biological systems. In order to allow comparison between different phyla and the robots any leg segments which are co-axial are counted as a single segment. This means that mammals are considered to have a single leg segment, and spiders are considered to have four.

It was found that for the most part the evolutionary algorithm results required less power to move a given payload; as the designs got larger less power was required per $\mathrm{kg}$ of payload, unlike the existing designs where larger designs were less efficient. The relative speed of the existing designs increased with the size of the robot; however for the evolutionary algorithm results the relative speed stayed approximately the same, at similar relative speeds to the slower existing designs. This could be due to the heavier existing designs having more powerful motors. Another reason could be that evolutionary algorithm results have more square bodies to minimize torque, however this means the legs are closer together, and thus the maximum speed of the system is lower. The smaller body does have the advantage that in most cases the evolutionary algorithm

TABLE 2

COMPARISON OF EXISTING DESIGNS AND EVOLUTIONARY PROGRAM

\begin{tabular}{|c|c|c|c|c|c|}
\hline \multicolumn{6}{|c|}{ RESULTS } \\
\hline & $\begin{array}{c}\text { Mass } \\
(\mathrm{kg})\end{array}$ & $\begin{array}{l}\text { Payload } \\
\text { mass } \\
\text { fraction }\end{array}$ & $\begin{array}{l}\text { Power } \\
\text { required } \\
\text { (W/ kg of } \\
\text { payload) }\end{array}$ & $\begin{array}{l}\text { Speed } \\
(\mathrm{BL} / \mathrm{s})\end{array}$ & $\begin{array}{l}\text { Work- } \\
\text { space }\end{array}$ \\
\hline $\begin{array}{l}\text { Boadicea } \\
{[14]}\end{array}$ & 4.90 & 0.47 & 3.91 & 0.22 & $0.003 *$ \\
\hline $\begin{array}{l}\text { Scorpion } \\
{[15]}\end{array}$ & 11.5 & 0.17 & $\begin{array}{l}\text { Up to } \\
36^{* *}\end{array}$ & 0.46 & $0.057 *$ \\
\hline $\begin{array}{l}\text { CAPTAIN } \\
{[16]}\end{array}$ & 23.0 & 0.087 & 4.00 & 0.37 & $0.167 *$ \\
\hline $\begin{array}{l}\text { ARAMIES } \\
{[17]}\end{array}$ & 28.0 & 0.18 & 4.98 & - & $0.073 *$ \\
\hline $\begin{array}{l}\text { Big Dog } \\
\text { [18] }\end{array}$ & 109.0 & 0.46 & 5600 & $\begin{array}{l}0.18- \\
1.82\end{array}$ & $1.30^{*}$ \\
\hline EP1 & 4.32 & 0.23 & 6.86 & 0.18 & 0.10 \\
\hline EP2 & 8.35 & 0.24 & 3.38 & 0.16 & 0.33 \\
\hline EP3 & 18.8 & 0.21 & 1.78 & 0.21 & 0.03 \\
\hline EP4 & 27.9 & 0.22 & 2.15 & 0.17 & 0.09 \\
\hline EP5 & 102.7 & 0.19 & 0.77 & 0.09 & 0.02 \\
\hline
\end{tabular}

* Estimated based on available leg dimensions

** Assuming three $6 \mathrm{~W}$ motors per leg, and a tetrapod gait
TABLE 3

COMPARISON OF BIOLOGICAL SYSTEMS AND EVOLUTIONARY PROGRAM RESULTS

\begin{tabular}{|c|c|c|c|c|c|c|}
\hline & \multirow{2}{*}{$\begin{array}{l}\text { Mass } \\
(\mathrm{kg})\end{array}$} & \multirow{2}{*}{$\begin{array}{l}\text { Number } \\
\text { of legs }\end{array}$} & \multicolumn{4}{|c|}{$\begin{array}{l}\text { Leg segments (relative to } \\
\text { segment } 1 \text { ) }\end{array}$} \\
\hline & & & 4 & 3 & 2 & 1 \\
\hline Insects [19] & $\begin{array}{l}0.025 \mathrm{~g} \\
-71 \mathrm{~g}\end{array}$ & 6 & 0.26 & 0.15 & 1.05 & 1 \\
\hline $\begin{array}{l}\text { Spiders* } \\
{[19]}\end{array}$ & $\begin{array}{l}0.053 \mathrm{~g} \\
-175 \mathrm{~g}\end{array}$ & 8 & 1.31 & 0.28 & 0.66 & 1 \\
\hline $\begin{array}{l}\text { Ground } \\
\text { dwelling } \\
\text { lizards [21] }\end{array}$ & $\begin{array}{l}0.12 \mathrm{~g}- \\
166 \mathrm{~kg}\end{array}$ & 4 & 0 & 0.95 & 0 & 1 \\
\hline $\begin{array}{l}\text { Cursorial } \\
\text { mammals } \\
{[21]}\end{array}$ & $\begin{array}{l}5 \mathrm{~kg}- \\
11,000 \\
\mathrm{~kg}\end{array}$ & 4 & 0 & 0 & 0 & 1 \\
\hline EP1 & 4.32 & 6 & 2.50 & 0.17 & 1.83 & 1 \\
\hline EP2 & 8.35 & 6 & 0.91 & 0.44 & 0.03 & 1 \\
\hline EP3 & 18.8 & 8 & 0.74 & 0.13 & 0.17 & 1 \\
\hline EP4 & 27.9 & 6 & 1.06 & 0.06 & 0.21 & 1 \\
\hline EP5 & 102.7 & 6 & 0.26 & 0.06 & 0.03 & 1 \\
\hline
\end{tabular}

*In reality, spiders have seven approximately equal leg segments; some of these have been combined to make comparison easier

results have a higher workspace than the existing designs.

In comparison with natural systems, it was found there were significant differences, for example the configurations of their legs suggest that torque is often not the most important factor, possibly because only one type of actuator is available (i.e. animal muscle, which is similar in all types of animal), and as long as the torque does not exceed the parameters of this actuator the actual torque requirement does not matter. However, most of the designs do have similar relative leg segment lengths to spiders, where segments 1 and 4 are the longest, and segment 2 is longer than segment 3 . The exceptions to this are the smallest design, EP1, in which segment 2 is longer than segment 1 , similarly to insect legs, and the largest design, EP5, for which segments 2, 3 and 4 are significantly smaller than segment 1 , which is similar to the way larger animals have compensated for their size by adopting more upright postures. The main mechanical factors which make animals successful are their ability to escape predators, and catch their food, both of which require speed and reach, thus their evolution leans towards these factors. However, for mobile robots, and especially robots for space exploration, torque is often the limiting factor in its performance. This suggests that copying a design directly from nature is not the best approach (for example by scaling up a cockroach or stick insect), unless the engineer specifically requires speed and workspace over torque minimization.

\section{Practicality of building the designs}

It was important that the algorithm produced designs which could be built using real world manufacturing techniques and materials. One safeguard to enable this was incorporated into the algorithm; the shear, tensile and compressive strengths of the selected material were compared against the stresses in the legs to ensure that the legs would not break. However some other factors had to be considered, for example the torque needed to lift the legs, which may be too high to achieve without using gearing. Related to this is the power required, since the rover will have limited power available. 
The designs considered are the results EP1-5. It can be seen in Figure 1 that even the lightest design EP1 required $4.64 \mathrm{Nm}$ of torque per leg, meaning that if the selected Faulhaber servo was used a gearbox with a 1:17 gearbox would be required, which might make the robot even slower. Gears are also susceptible to damage from dust and grit. This suggests that alternative actuators such as pneumatics might be useful for smaller robots which do not have the carrying capacity for large batteries, and indeed this was the strategy used in the Boadicea robot [14].

\section{CONCLUSIONS}

The algorithm discussed in this paper is able to produce results which require less power and lower torque motors than comparably sized existing robot designs. Additionally it produces designs which although not based on specific animals have similarities with biological systems, suggesting that these design features are of use both to robots and organisms. A number of design characteristics which could be applied to future robot designs were also found; it appears that a square or near square body has advantages in reducing power requirements, and that leg segment lengths should be equal for maximizing the workspace of a leg of a given length. Interestingly this concept is quite similar to the body configuration of spiders, whose legs are made up of seven equal segments, surrounding a single almost circular body. This suggests that spiders might be another useful model for roboticists to study, along with cockroaches and stick insects.

This algorithm can only produce bilaterally symmetric designs; however the fact that a near square (or circular) body has advantages suggests that rotationally symmetric systems might be worth investigating. As well as extending the algorithm to include rotational symmetry, it is planned to validate the algorithm by using it to design a robot chassis suitable for testing biomimetic controllers.

\section{REFERENCES}

[1] R. Altendorfer, N. Moore, H. Komsuoglu, M. Buehler, H. B. Brown Jr., D. McMordie, U. Saranli, R. Full, D. E. Koditschek, "RHex: A Biologically Inspired Hexapod Runner" Journal of Autonomous Robots 11, 207-213, 2001

[2] J. Ayers, "A Reactive Ambulatory Robot Architecture for Operation in Current and Surge" in Proc. of the Autonomous Vehicles in Mine Countermeasures Symposium, Naval Postgraduate School, Pp. 15-31 (1995)

[3] A. Asbeck, S. Dastoor A. Parness L Fullerton N. Esparza D. Soto B. Heyneman M. Cutkosky "Climbing rough vertical surfaces with hierarchical directional adhesion" in Proc. of the 2009 IEEE international conference on Robotics and Automation, Kobe, Japan, pp. 4328-4333 (2009)

[4] D. K. Pratihar, K. Deb, A. Ghosh "Design of a Genetic-Fuzzy System for Planning Optimal Path and Gait Simultaneously of a Six-legged Robot" In Proc. The Genetic and Evolutionary Computation Conference, Orlando, Florida, USA, July 13 - 17, 1999

[5] C. Paul, J. C. Bongard "The Road Less Travelled: Morphology in the Optimization of Biped Robot Locomotion" In Proc of the IEEE/RSJ International Conference on Intelligent Robots and Systems, 2001

[6] K. Sims "Evolving Virtual Creatures" Computer Graphics, Annual Conference Series, (SIGGRAPH '94 Proceedings), pp.15-22, July 1994

[7] K. S. Fu, R. C. Gonzalez, C. S. Lee C. S., Robotics: Control, Sensing, Vision and Intelligence McGraw- Hill Inc. 1987
[8] D. D. Ardayfio, Fundamentals of Robotics CRC Press 1987

[9] K. Waldron, V. Vohnout, A. Perry, R. McGhee, "Configuration design of the adaptive suspension vehicle" International Journal of Robotics Research 3 (2): 37-48 (1984)

[10] L. H. Ting, R. Blickhan, R. J. Full, "Dynamic and Static Stability in Hexapedal Runners" Journal of Experimental Biology 197: 251-269 (1994)

[11] E. F. Fichter, B. L. Fichter, "A Survey of Legs of Insects and Spiders From a Kinematics Perspective", in Proc. of the 1988 IEEE International Conference on Robotics and Automation, 2: 984 - 986, 24-29 Apr 1988

[12] S. Boettcher, A. G. Percus, "Optimization with Extremal Dynamics" Complexity 8(2) (2003)

[13] G.R. Wilson et al., "Mars Surface Mobility: Comparison of Past, Present, and Future Rover systems", 36th Annual Lunar and Planetary Science Conference, League City, Texas, March 14-18, 2005

[14] M. B. Binnard "Design of a Small Pneumatic Walking Robot" MS Thesis, Massachusetts Institute of Technology, MA, USA, January 2005

[15] B. Klaassen, R. Linnemann, D. Spenneberg, F. Kirchner, "Biomimetic walking robot SCORPION: Control and modelling" Journal of Robotics and Autonomous Systems 41 69-76 (2002)

[16] A. Ellery, G. P. Scott, P. Husbands, Y. Gao et. al., "Bionics \& Space Systems Design Case Study 1 - Mars Walker," Report Number: Contract AO/1-4469/03/NL/SFe, European Space Agency, Advanced Concepts Team 2005

[17] D. Spenneberg, A. Strack, J. Hilljegerdes, H. Zschenker, M. Albrecht, T. Backhaus, and F.Kirchner "ARAMIES: A four legged climbing and walking robot" in Proc. of 8th International Symposium iSAIRAS, Munich, Germany, September 5-8, 2005

[18] M. Raibert, K. Blankespoor, G. Nelson, R. Playter et al. "BigDog, the Rough-Terrain Quadruped Robot" in Proc. the 17th World Congress The International Federation of Automatic Control, Seoul, Korea, July 6-11, 2008

[19] E. F. Fichter, B. L. Fichter, "A survey of legs of insects and spiders from a kinematic perspective" in Proc. of the 1988 IEEE International Conference on Robotics and Automation, 2: 984 - 986, 24-29 Apr 1988.

[20] D. J. Irschick, B. C. Jayne "Comparative three- dimensional kinematics of the hind limb for high-speed bipedal and quadrupedal locomotion of lizards" The Journal of Experimental Biology 202: 1047-1065 (1999)

[21] S. Wroe, M. B. Lowry, M. Anton, "How to build a mammalian superpredator" Journal of Zoology 111: 196-203 (2008) 